\title{
Modes and Carrier Density in Dispersive and Nonlinear Gain Planar Photonic Crystal Cavity
}

\author{
Renlong Zhou, ${ }^{1}$ Mengxiong Wu, ${ }^{1}$ Yingyi Xiao, ${ }^{2}$ Lingxi Wu, ${ }^{1}$ Qiong Liu, ${ }^{1}$ Suxia Xie, \\ Hui Deng, ${ }^{1}$ Lisan Zeng, ${ }^{1}$ and Guozheng Nie ${ }^{1}$ \\ ${ }^{1}$ School of Physics and Electronic Science, Hunan University of Science and Technology, Xiangtan 411201, China \\ ${ }^{2}$ School of Foreign Studies, Hunan University of Science and Technology, Xiangtan 411201, China \\ Correspondence should be addressed to Renlong Zhou; rlzhou@mail.sitp.ac.cn
}

Received 24 January 2014; Accepted 9 March 2014; Published 17 April 2014

Academic Editor: Gong-Ru Lin

Copyright (C) 2014 Renlong Zhou et al. This is an open access article distributed under the Creative Commons Attribution License, which permits unrestricted use, distribution, and reproduction in any medium, provided the original work is properly cited.

The cavity mode and carrier density in dispersive and nonlinear gain planar photonic crystal cavities are studied with the threedimensional finite-difference time-domain method. Planar photonic crystal cavity can enhance light mater interaction, which can be used to design a photonic crystal cavity laser. With the effect of both total internal reflection and photonic band gap confinement, the frequency responses of the planar photonic crystal cavity can be obtained by simulation. The effect of carrier diffusion is calculated through the laser rate equations. The electric field intensity distribution, temporal behavior of electric field energy, and carrier density characteristics are analyzed from the resonance cavity mode.

\section{Introduction}

Many kinds of photonic crystal $(\mathrm{PhC})$ cavities have been studied [1-3]. The frequency responses of the planar photonic crystal defect structure can be studied with the effect of both total internal reflection and photonic band gap confinement. The effects of carrier diffusion in planar photonic crystal lasers have been studied using the nonlinear dispersive finitedifference time-domain (FDTD) method with solving the Maxwell equations in dispersive and nonlinear media [49]. The electrically driven $\mathrm{PhC}$ laser was analyzed with the FDTD method in studying interaction of light and matter [10]. The PhC microlaser analyses were studied with the FDTD method combined with the two-dimensional laser rate equation solver [11]. The three-dimensional finite-difference time-domain method that can handle dispersive and dynamic nonlinear gain media is proposed and realized in $\mathrm{PhC}$ laser cavity $[12,13]$. The design of photonic crystal semiconductor optical amplifier with polarization independence was sported [14]. The impulse response function has been extended based on the linear systems using a self-consistent timetransformation approach so that it can be applied to nonlinear media [15].
In our paper, the photonic crystal cavity structures including one and two cavities are studied. The photonic crystal vertical cavity surface emitting cavity is the light outputting region and is used to improve the outputting power of the modes. In order to study the dynamic interaction between the dispersive gain medium and electromagnetic fields in the $\mathrm{PhC}$ laser cavity, the dispersive nonlinear FDTD combined with auxiliary differential equations (ADE) method and the equation of carrier diffusion is applied. With the effect of both total internal reflection and photonic band gap confinement, the frequency responses of the planar photonic crystal cavity are studied. And the electric field intensity, temporal behavior of electric field energy, and carrier density characteristics are directly observed from the resonance modes.

\section{Nonlinear Dispersive Gain FDTD Method with ADE Method}

The nonlinear dispersive gain FDTD method has been used to a $\mathrm{PhC}$ laser $[12,13]$ and a semiconductor optical amplifier of $\mathrm{PhC}$ waveguides [14]. The relationship between the electric displacement $\vec{D}$ and the electric field $\vec{E}$ can describe the 


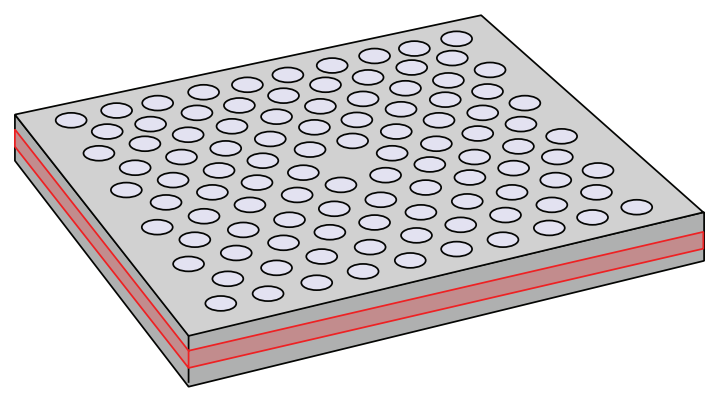

FIgure 1: The planar PhC cavity structure in the simulation. The red area layer is the dispersive nonlinear gain material and spatially uniform current pumping area in the $\mathrm{PhC}$ slab.

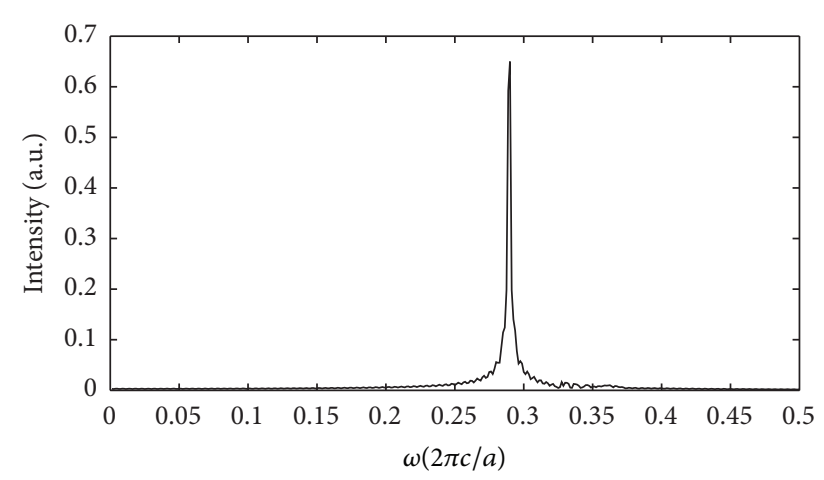

FIgURE 2: The frequency response of the planar PhC defect cavity.

different real material. Instead of having the relationship $\vec{D}=\varepsilon \vec{E}$ where $\varepsilon$ is a scalar constant, one can describe different behaviors of material regarding $\varepsilon$ as a function. The permittivity can also be written as a nonlinear function of the applied electric field to account for nonlinear media. The material parameters can also be functions of time. It should be noted that the permittivity can also be a function of position to describe spatial inhomogeneities. When the permittivity of a material is a function of frequency, the material is dispersive.

The nonlinear dispersive gain medium is approximated by the complex electric relative permittivity based on the classical model of the Lorentzian dipole oscillators and expressed by $\varepsilon_{r}(r, \omega)$. The optical property is difficult to be analytically described in the visible/near-UV region due to the interband transitions. In order to achieve a reasonable representation of the dielectric function, Etchegoin et al. [16] took inspiration from the parametric critical points model developed for semiconductors [17]. In this approach, the frequency dependence of the optical properties of nonlinear dispersive gain medium in the visible/near-UV region may be well described by an analytical formula with two main contributions that can be expressed as follows. Thus, we can write [12-14] the following:

$$
\begin{gathered}
\varepsilon_{r}(\vec{r}, \omega) \cong \varepsilon_{\infty}\left[1+\frac{\Gamma(\vec{r})}{\omega_{0}-\omega-i \gamma_{0}}+\frac{\Gamma(\vec{r})}{\omega_{0}+\omega+i \gamma_{0}}\right], \\
\Gamma(\vec{r}, t) \cong \frac{2 \gamma_{0} c n(\vec{r})}{\omega_{0}} G(N(\vec{r}, t) ; \vec{r}), \\
\frac{d N(\vec{r}, t)}{d t}=\frac{J(\vec{r}, t)}{q_{e}}-\frac{N(\vec{r}, t)}{\tau} \\
\quad+\frac{\vec{E}(\vec{r}, t)}{\hbar \omega_{0}} \frac{\partial \vec{P}(\vec{r}, t)}{\partial t}+D \nabla^{2} N(\vec{r}, t), \\
\frac{N(\vec{r}, t)}{\tau} \cong A N(\vec{r}, t)+B N^{2}(\vec{r}, t)+C N^{3}(\vec{r}, t), \\
-B N^{2}+\frac{\vec{E}(\vec{r}, t)}{\hbar \omega_{0}} \frac{\partial \vec{P}(\vec{r}, t)}{\partial t} \\
=\frac{\vec{E}(\vec{r}, t)}{\hbar \omega_{0}}\left[-\nabla \times \vec{H}(\vec{r}, t)+\varepsilon_{\infty} \frac{\partial \vec{E}(\vec{r}, t)}{\partial t}\right],
\end{gathered}
$$

where the resonance frequency and the damping constant are $\omega_{0}=2 \pi \times 193.41 \mathrm{THz}$ and $\gamma_{0}=1 \mathrm{THz}$, respectively, $\varepsilon_{\infty}$ is 11.9 , the $n(\vec{r})$ is real part of refractive index, $c$ is light velocity in vacuum, the material gain $G(N(\vec{r}, t) ; \vec{r})$ depends on the carrier density $N(\vec{r}, t)$ and position $\vec{r}$, the $G$ has the form $G(N(\vec{r}, t) ; \vec{r})=G_{0} \ln \left(N(\vec{r}, t) / N_{t r}\right), N_{t r}=$ $1.5 e^{19} \mathrm{~cm}^{-3}$ is transparent carrier density, the $G_{0}=1500 \mathrm{~cm}^{-1}$ is the linear gain constant, the $q_{e}$ and $\hbar$ are the elementary charge and the Planck constant, the $D=7.466 \mathrm{~cm}^{2} \mathrm{~s}^{-1}$ is the ambipolar carrier diffusion coefficient in $(3), J(r, t)$ is the pump-current density, and $\tau$ is the carrier life-time. The loss of the carrier density due to nonradiative recombination is shown in (4) with $A=3.5 e^{7} \mathrm{~s}^{-1}, B=1.6 e^{-10} \mathrm{~cm}^{3} \mathrm{~s}^{-1}$, and $C=5.0 e^{-29} \mathrm{~cm}^{6} \mathrm{~s}^{-1}$ being the coefficients due to surface recombination, spontaneous radiative recombination, and Auger recombination, respectively.

We can use two time-varying Maxwell equations to simulate the behaviors of electromagnetic waves in dispersive gain medium by applying nonlinear dispersive gain FDTD method. The two time-varying Maxwell equations are shown as follows:

$$
\begin{aligned}
& \nabla \times \vec{H}(\vec{r}, t)=\frac{\partial \vec{D}(\vec{r}, t)}{\partial t}, \\
& \nabla \times \vec{E}(\vec{r}, t)=-\mu \frac{\partial \vec{H}(\vec{r}, t)}{\partial t} .
\end{aligned}
$$




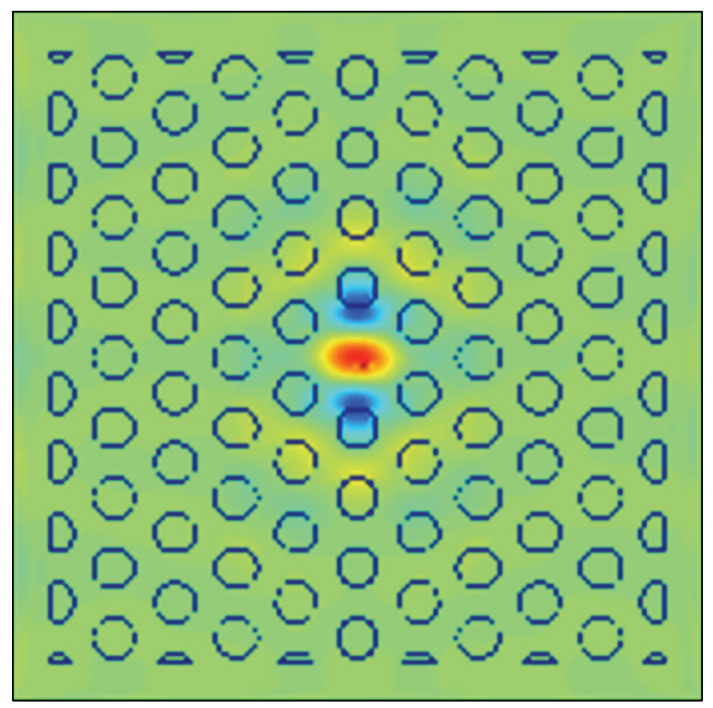

(a)

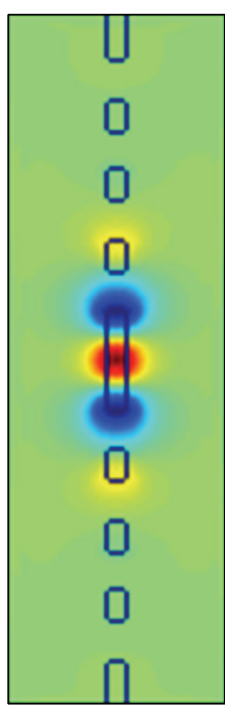

(b)

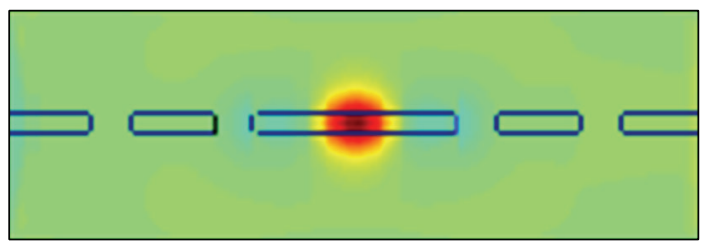

(c)

Figure 3: The distributions of electric field $E y$ for the resonance mode at 0.2895 are shown (a) in the $x y$ plane, (b) in the $y z$ plane, and (c) in the $z x$ plane.

The complex electric relative permittivity $\varepsilon_{r}(\vec{r}, \omega)$ can be used by introducing displacement vector

$$
\begin{gathered}
\vec{D}(\vec{r}, t)=\frac{1}{\sqrt{2 \pi}} \int_{-\infty}^{+\infty} \varepsilon_{0} \varepsilon_{r}(\vec{r}, \omega) \vec{E}(\vec{r}, \omega) e^{-i \omega t} d \omega, \\
\vec{D}(\vec{r}, \omega)=\varepsilon_{0} \varepsilon_{r}(\vec{r}, \omega) \vec{E}(\vec{r}, \omega),
\end{gathered}
$$

where $\vec{D}(r, \omega)$ and $\vec{E}(\vec{r}, \omega)$ are the Fourier coefficients of $\vec{D}(r, t)$ and $\vec{E}(r, t)$. form

To show the nonlocality in time, the term $\vec{D}(\vec{r}, t)$ has the

$$
\begin{aligned}
& \vec{D}(\vec{r}, t)=\varepsilon_{0} \varepsilon_{\infty}\left[\int_{-\infty}^{+\infty} d \tau g(\tau) \vec{E}(\vec{r}, t-\tau)+\vec{E}(\vec{r}, t)\right] \\
& g(\tau)=\frac{1}{2 \pi} \int_{-\infty}^{+\infty}\left[\frac{\varepsilon_{r}(r, \omega)}{\varepsilon_{\infty}}-1\right] e^{-i \omega \tau} d \omega \\
& =\frac{\Gamma(r)}{2 \pi} \int_{-\infty}^{+\infty}\left[\frac{1}{\omega_{0}-\omega-i \gamma_{0}}+\frac{1}{\omega_{0}+\omega+i \gamma_{0}}\right] e^{-i \omega \tau} d \omega .
\end{aligned}
$$

We can obtain the following equations from (1):

$$
\begin{gathered}
\vec{D}(\vec{r}, \omega)=\varepsilon_{0} \varepsilon_{\infty} \vec{E}(\vec{r}, \omega)+\varepsilon_{0} \varepsilon_{\infty} \beta(\vec{r}, \omega), \\
\beta(\vec{r}, \omega)=\left(\frac{\Gamma(r)}{\omega_{0}-\omega-i \gamma_{0}}+\frac{\Gamma(r)}{\omega_{0}+\omega+i \gamma_{0}}\right) \vec{E}(\vec{r}, w) .
\end{gathered}
$$

We obtain the following equation after rearranging (11):

$$
\begin{aligned}
& \omega_{0}^{2} \beta(\vec{r}, \omega)-\omega^{2} \beta(\vec{r}, \omega)+\gamma_{0}^{2} \beta(\vec{r}, \omega)-2 i \omega \gamma_{0} \beta(\vec{r}, \omega) \\
& \quad=2 \omega_{0} \Gamma(\vec{r}) \vec{E}(\vec{r}, \omega) .
\end{aligned}
$$

Then, taking the inverse Fourier transformation of (12),

$$
\begin{aligned}
\omega_{0}^{2} & \beta(\vec{r}, t)+\frac{\partial^{2}}{\partial t^{2}} \beta(\vec{r}, t)+\gamma_{0}^{2} \beta(\vec{r}, t)+2 \gamma_{0} \frac{\partial}{\partial t} \beta(\vec{r}, t) \\
& =2 \omega_{0} \Gamma(\vec{r}) \vec{E}(\vec{r}, t) .
\end{aligned}
$$

Representing (13) in terms of finite-differences,

$$
\begin{aligned}
\omega_{0}^{2} \beta^{n}(\vec{r}, t) & +\frac{\beta^{n+1}(\vec{r}, t)-2 \beta^{n}(\vec{r}, t)+\beta^{n-1}(\vec{r}, t)}{(\Delta t)^{2}} \\
& +\gamma_{0}^{2} \beta^{n}(\vec{r}, t)+2 \gamma_{0} \frac{\beta^{n+1}(\vec{r}, t)-\beta^{n-1}(\vec{r}, t)}{2(\Delta t)} \\
= & 2 \omega_{0} \Gamma(\vec{r}) E^{n}(\vec{r}, t) .
\end{aligned}
$$

Taking the inverse Fourier transformation of (10), we obtain

$$
\nabla \times \vec{H}(\vec{r}, t)=\varepsilon_{0} \varepsilon_{\infty} \frac{\partial \vec{E}(\vec{r}, t)}{\partial t}+\varepsilon_{0} \varepsilon_{\infty} \frac{\partial \vec{\beta}(\vec{r}, t)}{\partial t} .
$$

Expressing (15) for electric field in terms of finite-differences,

$$
\begin{aligned}
\vec{E}^{n+1}(\vec{r}, t)= & \vec{E}^{n}(\vec{r}, t)+\left(\frac{\Delta t}{\varepsilon_{0} \varepsilon_{\infty}}\right)\left(\nabla \times \vec{H}^{n+1 / 2}(\vec{r}, t)\right) \\
& -\left(\vec{\beta}^{n+1}(\vec{r}, t)-\vec{\beta}^{n}(\vec{r}, t)\right) .
\end{aligned}
$$

And the similar updated equation for magnetic field in terms of finite-differences is as follows:

$$
\vec{H}^{n+1 / 2}(\vec{r}, t)=\vec{H}^{n-1 / 2}(\vec{r}, t)-\frac{\Delta t}{\mu}\left(\nabla \times \vec{E}^{n}(\vec{r}, t)\right) .
$$




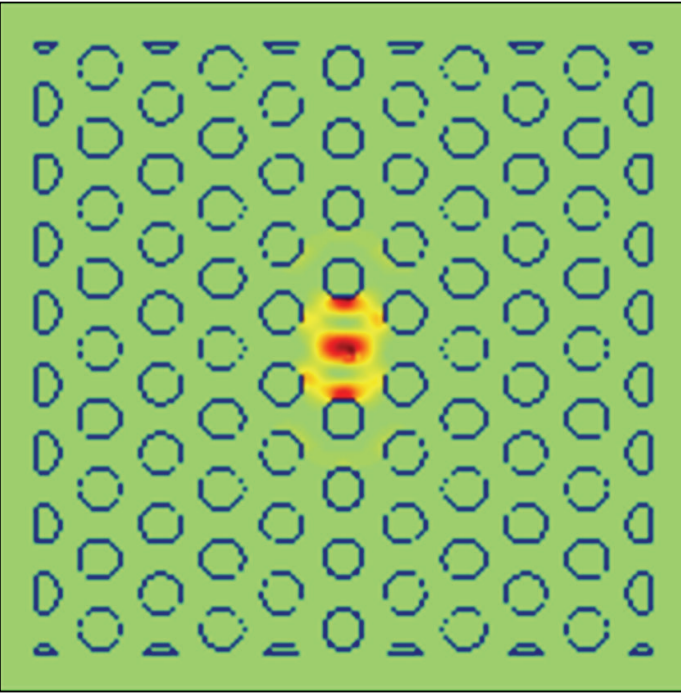

(a)

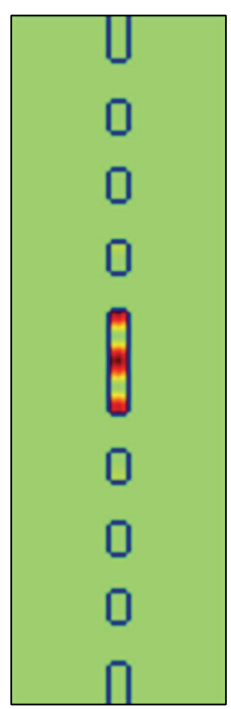

(b)

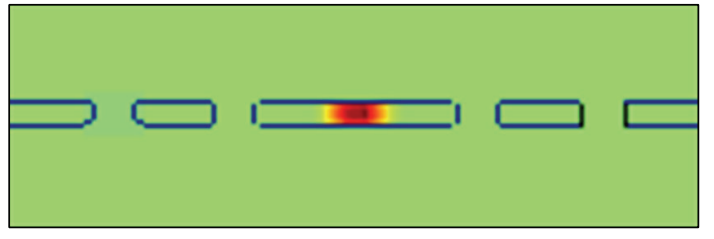

(c)

FIGURE 4: The distributions of carrier density for the resonance mode at 0.2895 are shown (a) in the $x y$ plane, (b) in the $y z$ plane, and (c) in the $z x$ plane.

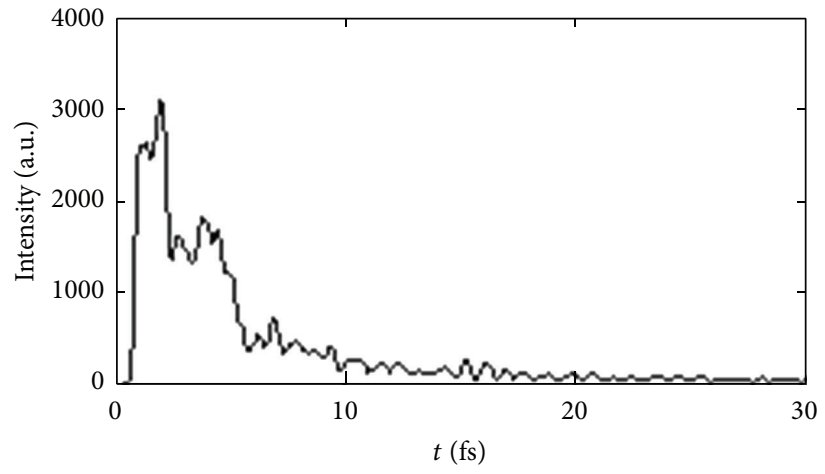

(a)

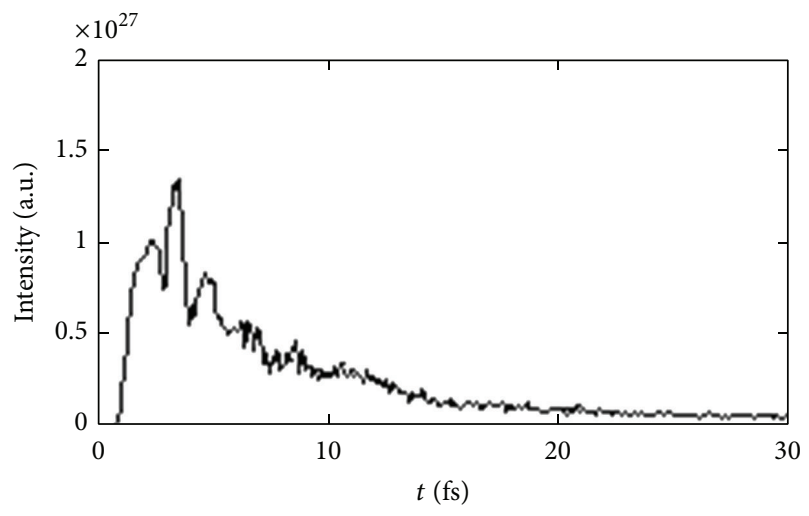

(b)

FIGURE 5: (a) The temporal behavior of the $|E|^{2}$ and (b) carrier density of the planar PhC defect cavity for the pulse incident wave.

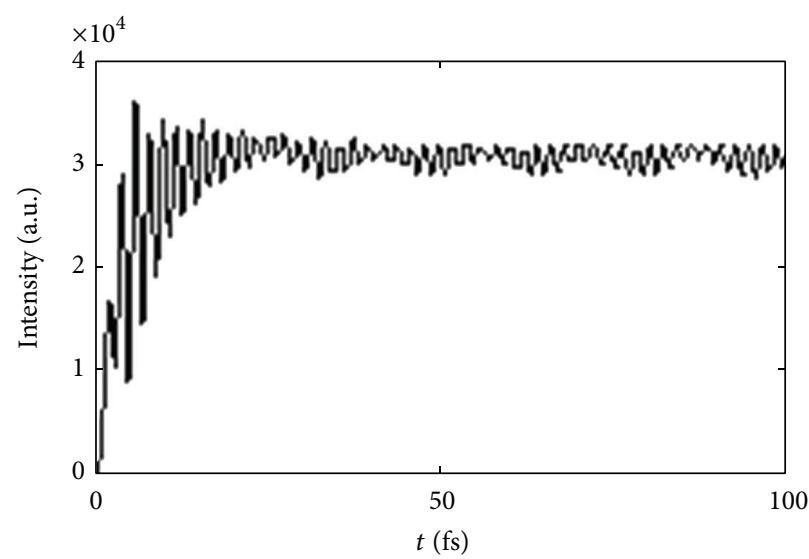

(a)

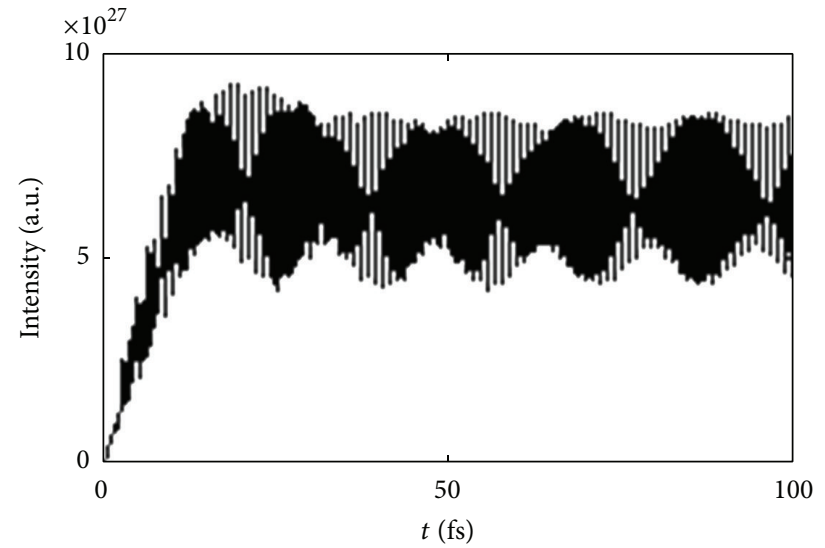

(b)

Figure 6: (a) The steady-state behavior of the $|E|^{2}$ and (b) carrier density of PhC defect cavity for continuous incident wave at the resonance frequency 0.2895 . 


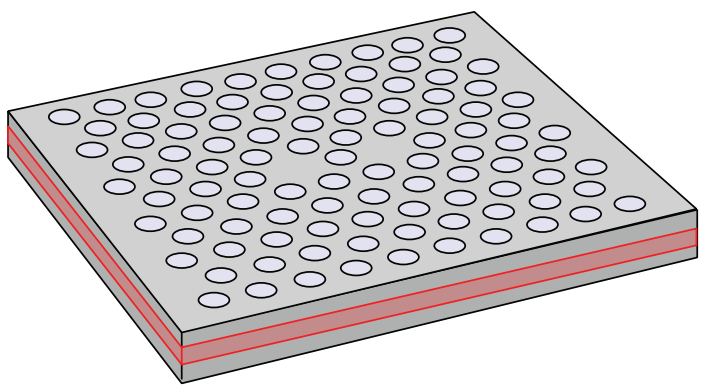

(a)

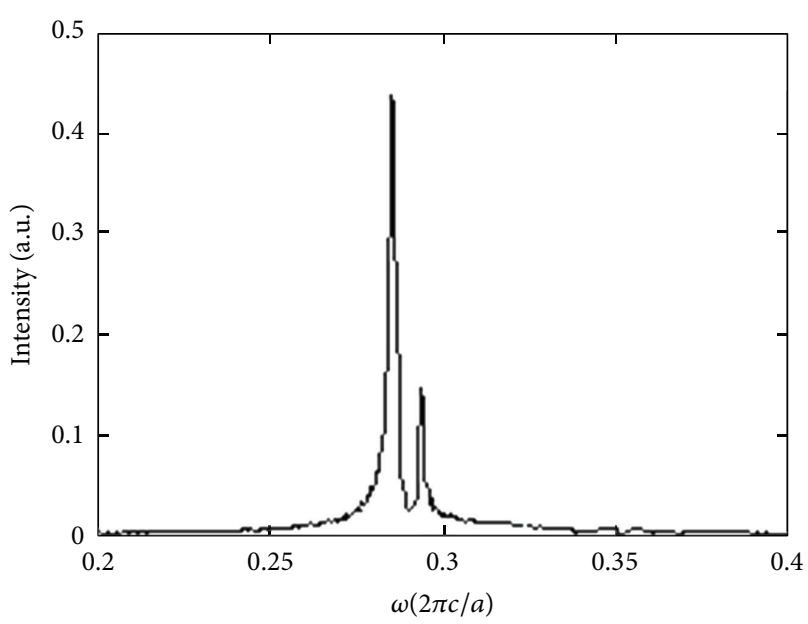

(b)

FIGURE 7: (a) The PhC cavity structure in the simulation. The red area layer is the nonlinear gain region and spatially uniform current pumping area in the PhC slab. (b) The frequency response of the PhC defect cavity at frequencies 0.283 and 0.2928.

\section{Simulation Results of the PhC Cavity}

Figure 1 shows the structure of a planar photonic crystal defect cavity which is created by a missing air hole in the photonic crystal slab with triangular lattice arrays along the $x y$ direction. The basic unit size of a $\mathrm{PhC}$ is the length of the period, which is usually called lattice constant $a$. The radius of air holes and the thickness of the PhC slab are $0.29 a$ and $0.6 a$, respectively. The dielectric constant of the dielectric slab material is assumed to be 11.56.

The red sandwich layer is the dispersive nonlinear gain medium that is buried in the $\mathrm{PhC}$ slab as shown in Figure 1. The intrinsic carrier density $1.5 \times 10^{12} \mathrm{~cm}^{-3}$ is used as the initial carrier density in the beginning $(t=0)$. The intensity of injection current is $5 e^{16} \mathrm{~A} / \mathrm{m}^{3}$. The injection current is uniformly supplied over the circular region of radius $2.5 a$ inside the red sandwich layer around the center of cavity.

Because the structure shows a TE-like photonic band gap from 0.256 to 0.32 in normalized frequency $(\omega a / 2 \pi c)$ [17], we use an initial TE-polarized electric field to excite the TElike mode of the defect structure. A point pulse source with the electric field originated in the $y$ direction is placed at the center of the defect cavity. In Figure 2, the frequency response of the PhC defect cavity at 0.2895 is located inside photonic band gap.

The intensity distributions of electric field Ey for the defect mode at 0.2895 are shown (a) in the $x y$ plane, (b) in the $y z$ plane, and (c) in the $z x$ plane in Figure 3. The intensity distributions of carrier density for the defect mode at 0.2895 are shown (a) in the $x y$ plane, (b) in the $y z$ plane, and (c) in the $z x$ plane in Figure 4 . The cavity mode preferentially consumes the carriers shaping up the region of the strong electric field with a characteristic carrier density profile.

To study the temporal development of the cavity mode, the energy of electric field in the cavity mode and the carrier density are computed with the result plot given in Figure 5. (a) The temporal behavior of the $|E|^{2}$ and (b) carrier density of the $\mathrm{PhC}$ defect cavity for the pulse incident wave are shown in Figure 5. It is also interesting to observe the relaxation oscillation after the onset of the cavity mode.

This oscillation gradually decays out. Compared to the temporal behavior, (a) the steady-state behavior of the $|E|^{2}$ and (b) carrier density for the continuous incident wave are also shown in Figure 6. The steady-state oscillation appears after $12 \mathrm{fs}$ for the continuous incident wave. It is expected that both the energy of electric field and the carrier density approach their steady-state values in $12 \mathrm{fs}$.

Figure 7(a) shows the structure of a planar photonic crystal defect cavity which is created by two missing air holes in the photonic crystal slab. The red area layer is the nonlinear gain region and spatially uniform current pumping area in the PhC slab. The frequency response of the two PhC defect cavities shows that there exist two defect resonance modes at frequencies 0.283 and 0.2928 within the photonic band gap as shown in Figure 7(b). The intensity distributions of electrical field $E y$ and carrier density with frequencies 0.283 and 0.2928 in the simulation of FDTD are shown in Figures $8(a)-8(d)$, respectively. The intensity distributions of electric field $E y$ for the two resonance modes at frequencies 0.283 and 0.2928 in the $x y$ plane are shown in Figures 8(a) and 8(c). The intensity of the lower frequency mode at frequency 0.283 is much stronger than that of the higher frequency mode at frequency 0.2928 .

\section{Conclusions}

In summary, we have used the three-dimensional FDTD method for the numerical simulation of cavity modes in dispersive and nonlinear gain planar photonic crystal cavity. Using this method, we have studied the frequency responses of the planar photonic crystal cavity. The study includes the distribution of the electric field intensity. Furthermore, the carrier density characteristics are also obtained from the laser rate equations. 


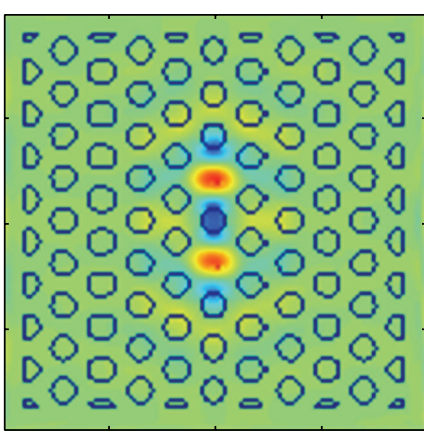

(a)

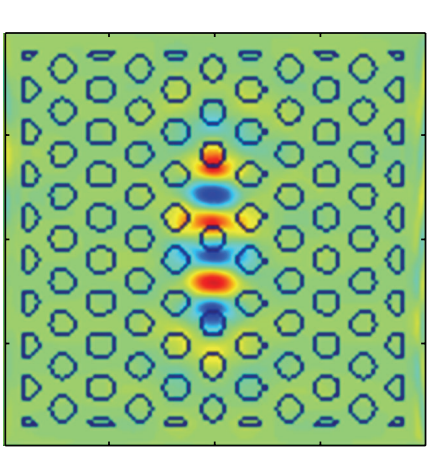

(c)
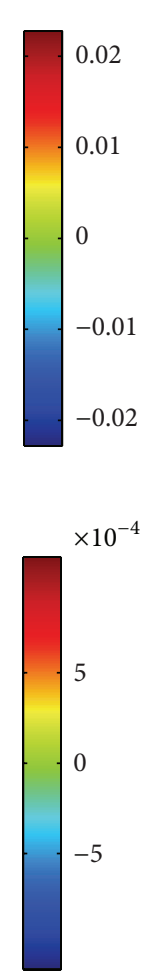$$
\text { . }
$$

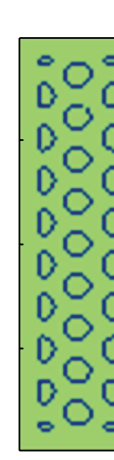

FIGURE 8: (a) The intensity distributions of electric field $E y$ and (b) carrier density at frequency 0.283. (c) The intensity distributions of electric field $E y$ and (d) carrier density at frequency 0.2928.

\section{Conflict of Interests}

The authors declare that there is no conflict of interests regarding the publication of this paper.

\section{Acknowledgments}

This work was supported by the National Natural Science Foundation of China under Grants nos. 11247003, 51175172, and 11304094, the Open Research Fund of National Laboratory for Infrared Physics (GJKF20130027 and GJKF20130028), the Chinese Academy of Sciences, and the Scientific Research Fund of Hunan Provincial Education Department (12C0143 and 13C323). The authors are most grateful to Yejin Zhang for stimulating discussions [14].

\section{References}

[1] O. Painter, R. K. Lee, A. Scherer et al., "Two-dimensional photonic band-gap defect mode laser," Science, vol. 284, no. 5421, pp. 1819-1821, 1999.

[2] H. Park, J. Hwang, J. Huh, H. Ryu, Y. Lee, and J. Kim, "Nondegenerate monopole-mode two-dimensional photonic band gap laser," Applied Physics Letters, vol. 79, no. 19, pp. 30323034, 2001.

[3] H. Y. Ryu, S. H. Kim, H. G. Park, J. K. Hwang, Y. H. Lee, and J. S. Kim, "Square-lattice photonic band-gap single-cell laser operating in the lowest-order whispering gallery mode," Applied Physics Letters, vol. 80, no. 21, pp. 3883-3885, 2002.

[4] A. Taflove, Computational Electrodynamics: The Finite-Difference Time-Domain Method, chapter 5, 8, 11, Artech House, Boston, Mass, USA, 1995.

[5] N. Guo, W.-D. Hu, X.-S. Chen, L. Wang, and W. Lu, "Enhanced plasmonic resonant excitation in a grating gated field-effect transistor with supplemental gates," Optics Express, vol. 21, no. 2, pp. 1606-1614, 2013.

[6] L. Wang, W. Hu, J. Wang et al., "Plasmon resonant excitation in grating-gated AlN barrier transistors at terahertz frequency," Applied Physics Letters, vol. 100, no. 12, Article ID 123501, 2012.

[7] W. D. Hu, L. Wang, X. S. Chen et al., "Room-temperature plasmonic resonant absorption for grating-gate GaN HEMTs in far infrared terahertz domain," Optical and Quantum Electronics, vol. 45, no. 7, pp. 713-720, 2013.

[8] G. Li, X. Chen, B. Ni et al., "Fractal H-shaped plasmonic nanocavity," Nanotechnology, vol. 24, no. 20, Article ID 205702, 2013.

[9] G. Li, X. Chen, O. Li et al., "A novel plasmonic resonance sensor based on an infrared perfect absorber," Journal of Physics D: Applied Physics, vol. 45, no. 20, Article ID 205102, 2012.

[10] H. G. Park, S. H. Kim, S. H. Kwon et al., "Electrically driven single-cell photonic crystal laser," Science, vol. 305, no. 5689, pp. 1444-1447, 2004.

[11] K. Nozaki and T. Baba, "Carrier and photon analyses of photonic microlasers by two-dimensional rate equations," IEEE Journal on Selected Areas in Communications, vol. 23, no. 7, pp. 1411-1416, 2005. 
[12] M. Fujita, A. Sakai, and T. Baba, "Ultrasmall and ultralow threshold GaInAsP-InP microdisk injection lasers: design, fabrication, lasing characteristics, and spontaneous emission factor," IEEE Journal on Selected Topics in Quantum Electronics, vol. 5, no. 3, pp. 673-681, 1999.

[13] M.-K. Seo, G. H. Song, I. Hwang, and Y. Lee, "Nonlinear dispersive three-dimensional finite-difference time-domain analysis for photonic-crystal lasers," Optics Express, vol. 13, no. 24, pp. 9645-9651, 2005.

[14] Y. Zhang, W. Zheng, Q. Aiyi et al., "Design of photonic crystal semiconductor optical amplifier with polarization independence," Journal of Lightwave Technology, vol. 28, no. 22, Article ID 5604633, pp. 3207-3211, 2010.

[15] Y. Xiao, D. N. Maywar, and G. P. Agrawal, "New approach to pulse propagation in nonlinear dispersive optical media," Journal of the Optical Society of America B, vol. 29, no. 10, pp. 2859-2963, 2012.

[16] P. G. Etchegoin, E. C. Le Ru, and M. Meyer, "An analytic model for the optical properties of gold," Journal of Chemical Physics, vol. 125, no. 16, Article ID 164705, 2006.

[17] P. Etchegoin, J. Kircher, and M. Cardona, "Elasto-optical constants of Si," Physical Review B, vol. 47, no. 16, pp. 10292-10303, 1993. 

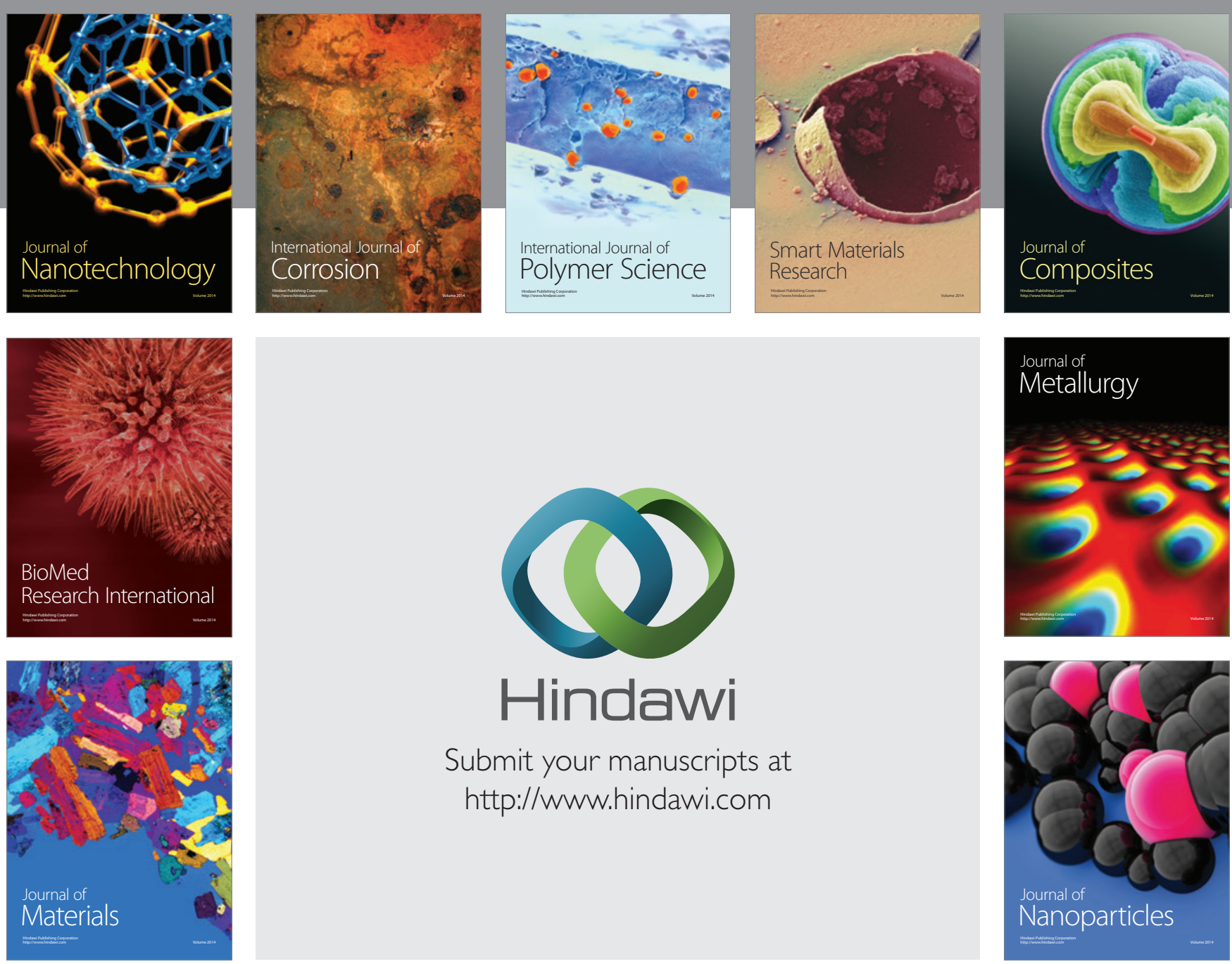

Submit your manuscripts at http://www.hindawi.com
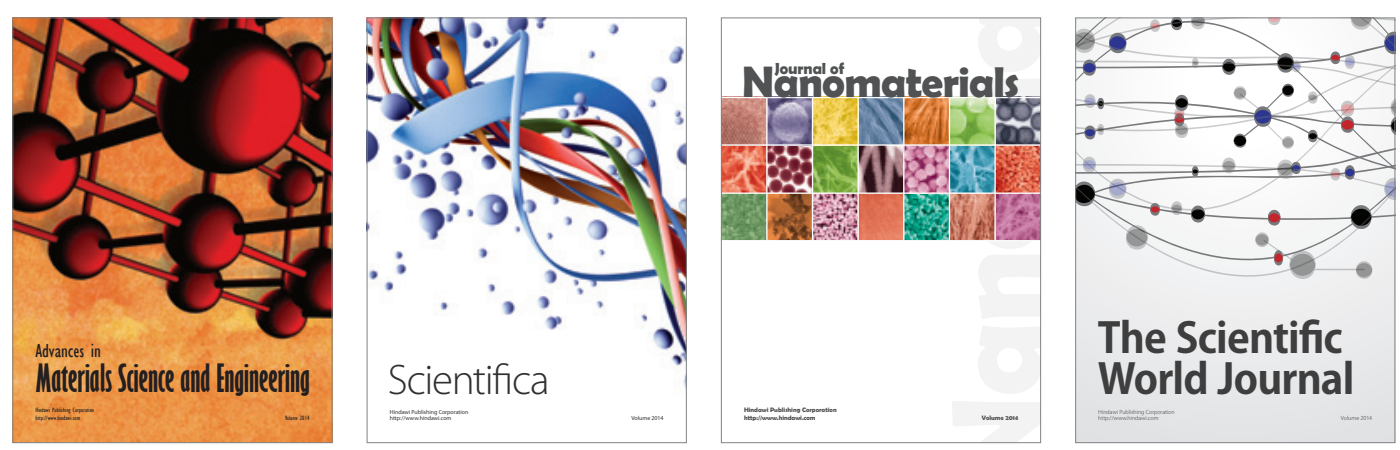

\section{The Scientific World Journal}
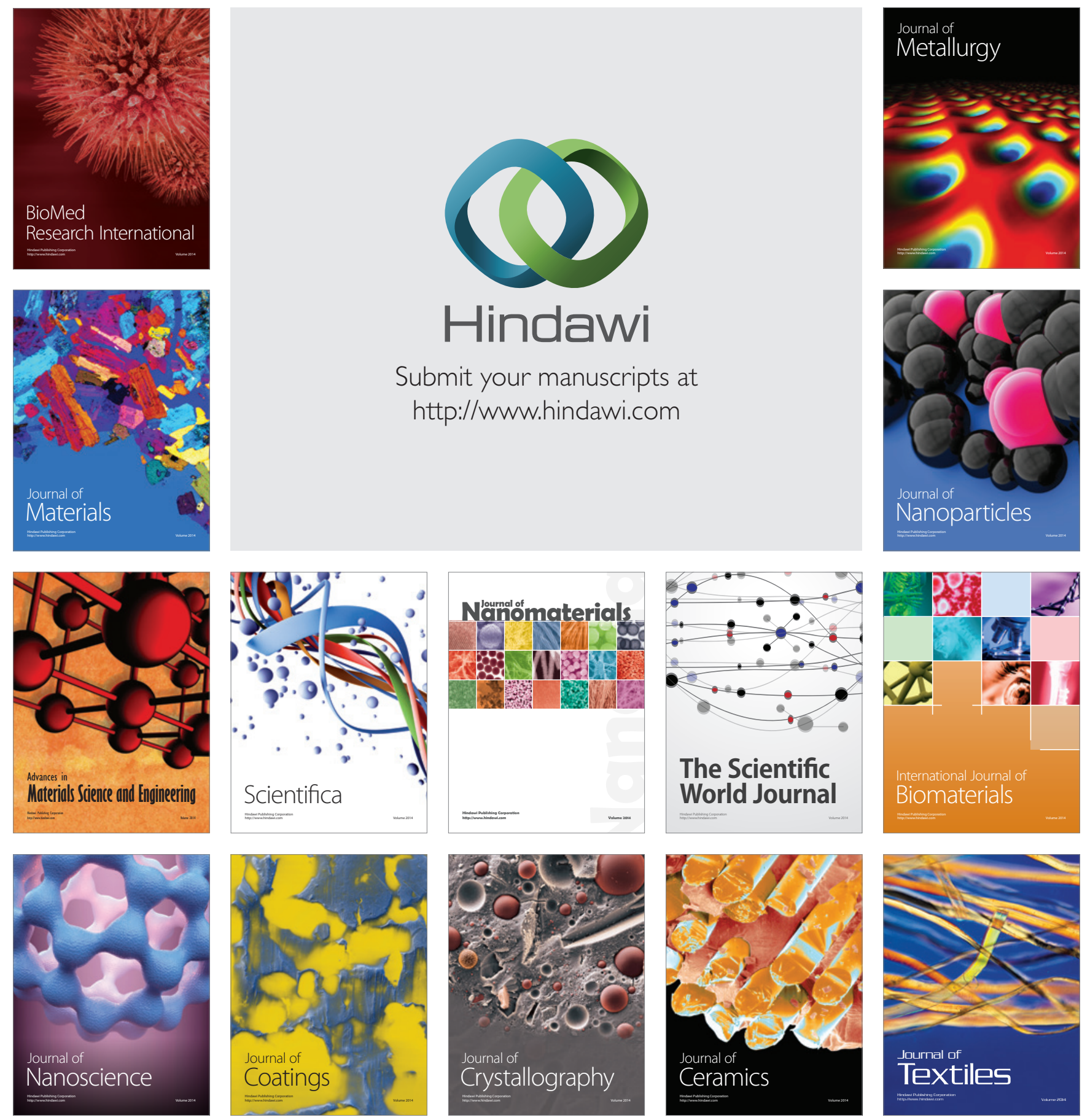